\title{
Conducting polymer: silver interface, morphology and properties
}

\author{
Jacek Nowaczyk $^{1}{ }^{1} \cdot$ Kornelia Kadac $^{1} \cdot$ Iwona $_{\text {Tarach }}{ }^{1} \cdot$ Ewa Olewnik-Kruszkowska $^{1}$
}

Received: 25 July 2017 / Accepted: 4 September 2017 / Published online: 7 September 2017

(c) The Author(s) 2017. This article is an open access publication

\begin{abstract}
Polymers having conjugated double bond system in the backbone structure are electronically conducting. Incorporation of certain side groups to such backbone gives introduce unique new properties or alter the initial properties of the polymer. Consequently conducting polymers with unique and tailored properties are valuable new materials of the future engineering. Electrical properties of the interface between conducting polymer and metal is of vital importance to the application potential of the polymer in the electronics. In present study we discuss the electrical properties of the heterojunction composed of conductive polymer pressed nanoparticles and silver thin layer. The contacts were deposited by vacuum evaporation from bulk silver plate or dry silver nanoparticles. In this study the new conjugated polymer poly(4-(thien-3-ylmethoxy)butane-1-sulfonic acid)—PTMBSA — was used as the organic semiconductor. The syntheses of PTMBSA and silver nanoparticles are discussed. In order to evaluate the morphology of PTMBSA pellets with electrodes scanning electron microscopy (SEM) was applied. The important information on the electrical properties of the system were obtained from the measurements of the current-voltage characteristics revealing significant qualitative difference of investigated heterojunctions. It was found that the contact of silver and PTMBSA has electrical properties varying in time. For about $100 \mathrm{~h}$ after preparation, intrinsic conductivity of the sandwich specimen has been gradually increasing.
\end{abstract}

Jacek Nowaczyk

jacek.nowaczyk@umk.pl

1 Faculty of Chemistry, Nicolaus Copernicus University in Toruń, Gagarina St. 7, 87-100 Toruń, Poland

\section{Introduction}

Conductive polymers are a group of macromolecular compounds, that combine the properties of conventional plastics with intrigue electrical properties of semiconductors. This combination make these polymers attractive in several applications [1-3]. Conductive polymers can conduct electricity according to different mechanisms. Three main mechanisms have been distinguished: electronic conductivity, ionic conductivity, and the redox conductivity. Consequently, conducting polymers can be arranged within three categories including specific molecular structures responsible for the charge transport. Electronic conductors include molecules containing delocalized (conjugated) $\pi$-electron systems similar to these in polyacetylene backbone. Ioniocally conducting polymers, also known as polyelectrolytes, contain ionophor group capable of electrolytic dissociation or heteroatoms with electron lone pairs that can contribute in solvation of external ions. The third group include polymers having pendant red/ox active groups that can reversibly change the oxidation state such as ferrocene or fullerenes [4]. Within these three groups electronic conductors are excellent candidates for application in microelectronics including manufacturing of devices such as filed effect transistors, organic light emitting diodes, photovoltaic cells or sensors $[5,6]$. The list of application is substantially longer, however these above mentioned share on important feature, they utilize the unusual property of interface between conducting polymer and metals or semiconductors. Especially the application in sensors manufacturing is of great importance. Conducting polymers have been demonstrated general analytical transduction performance that is of great demand in electrochemical sensors technology [7-9]. The source of high adaptability of conducting polymers in sensors technology consist in unique properties of these materials such as 
the flexibility, tunable electrical properties, and ability to undergo a wide range of molecular interactions. Especially the interactions that can alter a certain properties of these polymers are of great importance to application of conducting polymers as analytical transducer of biosensors [10]. Electronically conducting polymers are often synthesized insitu on the electrode surface using electrochemical deposition techniques [11]. The fecile nature of this technique make it possible to control thickness of the polymer layer as well as spatial distribution of biological compounds immobilized within the polymer matrix [12-14]. Up to date wide range of different organic semiconductors including conductive polymers was studied in variety of multilayer structures to investigate their electrical properties under heterojunction effect [15]. The insight in the intrinsic charge carrier transport is important to understand the interaction between organic semiconductors and working mechanism of a heterojunction based devices.

Polythiophene and its derivatives are among the most studied conductive polymers. These compounds are characterized by significant environmental and thermal stability, high conductivity, and small band gap values [16-19]. Chemical structure of polythiophene permits wide range of modification without destruction of conjugated double bond system responsible for the unique electronic properties $[20,21]$. The most common is introduction of specific side chain at $\beta$ carbon of thiophene ring. The major drawback of polythiophene based materials comes from the difficulty in processing. Most of polythiophene derivatives are insoluble in common solvents and do not fuse. To overcome this problem polymer composite materials and copolymerization have been studied. Recently synthesis of thiophene co-polymers with processable polymers was found to be interesting alternative to introduce valuable properties of conducting polymers to wider use [22, 23]. It was found that the introduction of alkyl side chain of specific length results in the increase of solubility and processability of polythiophene [19]. On the other hand derivatives containing ionophore group such as carboxyl, sulphonic or tertiary ammine groups, can make the polymer water soluble [24]. In addition another mechanism of charge carriers can be expected in such materials, consist of free ion migration. Incorporation of the side chains having ionic groups gives rise to new properties specific for polyelectrolytes. Polymeric materials that combine electrical conductivity of semiconductors with ionic conductivity of electrolytes are regarded to as conjugated polyelectrolytes [25-28].

In present work we study electrical property of metal semiconductor junction in the new polymer material obtained via emulsion polymerization of sulfonate group bearing thiophene derivative. Although some of the heterojunction behaviors remains similar for most of conducting polymers unceasing scientific and technological research is conducted to provide detailed information that can open the way for new and valuable applications.

In electronic application the most important properties of organic semiconductors are related to interface present at the contact of organic material and metal. Distribution of chemical potential in such interface is the source of specific properties such as ohmic conduction or charge refining. Bringing two solids having different band structures in to contact build up non equilibrium conditions. Such system tend to achieve the thermodynamic equilibrium through charge transfer between phases. As a consequence the bands are being bend and interfacial electric dipole layer equalize the chemical potentials. This dipole layer affect the current flow through the heterojunction.

In this study he have made an effort to investigate the properties of interface between new conjugated polyelectrolyte PTMBSA and silver. The silver was chosen as cheaper alternative to gold, since both the metals have high work function. Respectively, the heterojunction was expected to have ohmic rather than refining character. Moreover, in the course of the study it occurred that it make a significant difference if the $\mathrm{Ag}$ contacts were prepared from bulk silver plate or from silver nanoparticles. The aim of the study is to reveal and discuss the differences in heterojunction properties when it is formed of bulk metal or metal nanoparticles.

Another important aspect of this work is the application of emulsion polymerization of conducting polymers that is relatively new approach to conducting polymer synthesis with the green chemistry connotations [29-36].

\section{Experimental}

\subsection{Materials and methods}

Silver nitrate (99.9\%), potassium hydroxide (85\%), ammonia (25\%), D-glucose (96\%), hydrochloric acid (35\%), sodium hydride (60\% in mineral oil) and dimethylformamide (99.8\%) were obtained from Avantor Performance Materials Poland S.A., Gliwice, Poland. This reagents were used directly, without further purification. Silver (99.99\%) was purchased from Mennica - Metale Szlachetne, Radzymin, Poland.

3-thiophenemethanol (98\%), 1,4-butane sultone ( $\geq 99 \%$ ), sodium dodecyl sulfate ( $\geq 99 \%)$ and potassium peroxodisulfate ( $\geq 99 \%)$ and were purchased from Sigma-Aldrich Chemie GmbH, Schnelldorf, Germany. This reagents were used without further purification. Deionized water was used.

The XRD study was made using HZG-4/A-2 apparatus. Samples in form of powders were subjected to X-ray radiation in the angular range of $30^{\circ}-120^{\circ}$.

The morphology of nanosilver was examined using the transmission electron microscope (TEM) Tecnai F20 
X-Twin. The imaging of polymer surface with silver or nanosilver was performed using the atomic force microscope (AFM) NanoScope MultiMode SPM System and the scanning electron microscope (SEM) Quanta 3D FEG.

The electrochemical properties of polymer/metal heterojunction were analyzed for specimens consist of polymer pressed powder pellets covered with metal contacts. The pellets were obtained by pressing thoroughly ground polymer in the laboratory press under pressure 70 bar at room temperature. The silver and nanosilver electrodes were evaporated in vacuum $\left(1 \times 10^{-4}\right.$ torr $)$ above $1200{ }^{\circ} \mathrm{C}$. The thickness of the pellets was detrmined using the electronic thickness gauge SYLVAC.

The electrical properties of the heterojunctions was determined by means of current-voltage characteristics (i-v) and volume dc-conductivity $\left(\sigma_{\mathrm{dc}}\right)$ applying two point probe electrometer Keytley $6517 \mathrm{~B}$. The pellets were analyzed in dry state at 298, 308 and $318 \mathrm{~K}$. In order to study the influence of metal contacts stability measurements were repeated several times in time.

\subsection{The preparation of nanosilver particles}

Recently there are known different techniques of silver nanoparticle preparations [37], however in this work we have applied the method developed in our lab several years ago. The preparation procedure is a follows. Silver nitrate (30 mmol) was dissolved in deionized water $(0.8 \mathrm{~L}$ and placed in an ice bath for $1 \mathrm{~h}$ ). Afterwards $100 \mathrm{~cm}^{3}$ of $0,1 \mathrm{M}$ aqueous solution of potassium hydroxide $(100 \mathrm{mmol})$ was added to the mixture was then stirred and $40 \mathrm{~cm}^{3}$ of ammonia solution (25\%) was slowly introduced. Further, the temperature of the mixture and its temperature was elevated to $283 \mathrm{~K}$. Then, $70 \mathrm{~cm}^{3}$ of D-glucose solution (34 mmol) was poured in to the mixture which was left for about hour of continuous stirring. After that $4 \mathrm{dm}^{3}$ of distilled water was added followed by additional of second portion of reducing agent (glucose $34 \mathrm{mmol}$ ). Then the mixture was left to level the temperature to ambient conditions. Finally, the solution was mixed and kept in room temperature for $1 \mathrm{~h}$. The product was filtrated, washed thoroughly with deionized water and desiccated under vacuum at $40{ }^{\circ} \mathrm{C}$ for $48 \mathrm{~h}, 93 \%$ yield.

\subsection{The preparation of the organic semiconductor (PTMBSA)}

The synthesis of conducting polymer is a multistage process which was studied and optimized in our lab which was described in separate article [38]. In the first stage 3-thiophenemethanol (22 mmol) was dissolved in $100 \mathrm{ml}$ of dimethylformamide at $283 \mathrm{~K}$ then, sodium hydride (30 mmol) was added. The mixture was stirred at room temperature for $24 \mathrm{~h}$. 1,4-butane sultone ( $22 \mathrm{mmol})$ was introduced in the mixture and the solution was mixed for $24 \mathrm{~h}$. Afterwards the solvent was evacuated and resulting 4-(thien-3-ylmethoxy)butane-1-sulfonic acid (TMBSA) was dried, $76 \%$ yield (Fig. 1).

In the second stage, poly(4-(thien-3-ylmethoxy)butane1-sulfonic acid) (PTMBSA) was prepared by emulsion polymerization. TMBSA (50 mmol) and sodium dodecyl sulfate (SDS, $25 \mathrm{mmol})$ was dissolved in water $\left(30 \mathrm{~cm}^{3}\right.$, $1660 \mathrm{mmol}$ ). Then, potassium peroxodisulfate (KPS, $50 \mathrm{mmol})$ was dissolved in water $\left(40 \mathrm{~cm}^{3}, 2213 \mathrm{mmol}\right)$. The polymerization was initiated by dropwise addition of KPS solution into preheated reaction mixture $\left(70{ }^{\circ} \mathrm{C}\right)$. The process was carried out for $5 \mathrm{~h}$ and then terminated by pouring the emulsion into methanol $\left(500 \mathrm{~cm}^{3}\right)$. The product was collected by filtration and washed thoroughly with methanol $\left(150 \mathrm{~cm}^{3}\right)$ and with water $\left(150 \mathrm{~cm}^{3}\right)$. The powder was dried, $63 \%$ yield (Fig. 2 ).

\section{Results and discussion}

\subsection{X-ray diffraction study}

X-ray diffraction patterns of powder silver nanoparticles are shown in Fig. 3. The presence of peaks at $2 \theta$ values $38.29^{\circ}, 44.47^{\circ}, 64.60^{\circ}, 77.55^{\circ}, 81.68^{\circ}, 98.01^{\circ}, 110.63^{\circ}$ and $115.04^{\circ}$, corresponding with the following Miller Indices
Fig. 1 The scheme of the monomer 4-(thien-3-ylmethoxy) butane-1-sulfonic acid synthesis
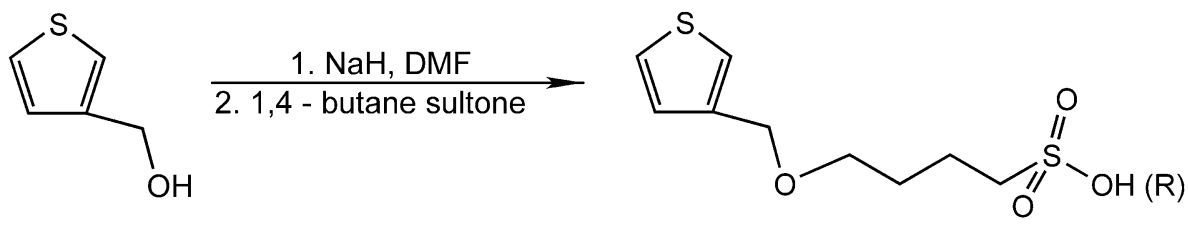

Fig. 2 The scheme of the polymer synthesis
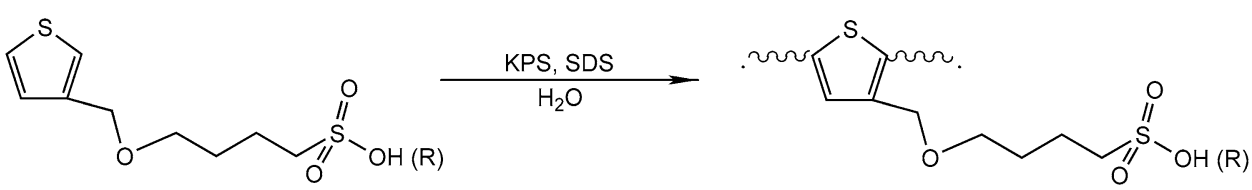
Fig. 3 XRD pattern of silver nanoparticles

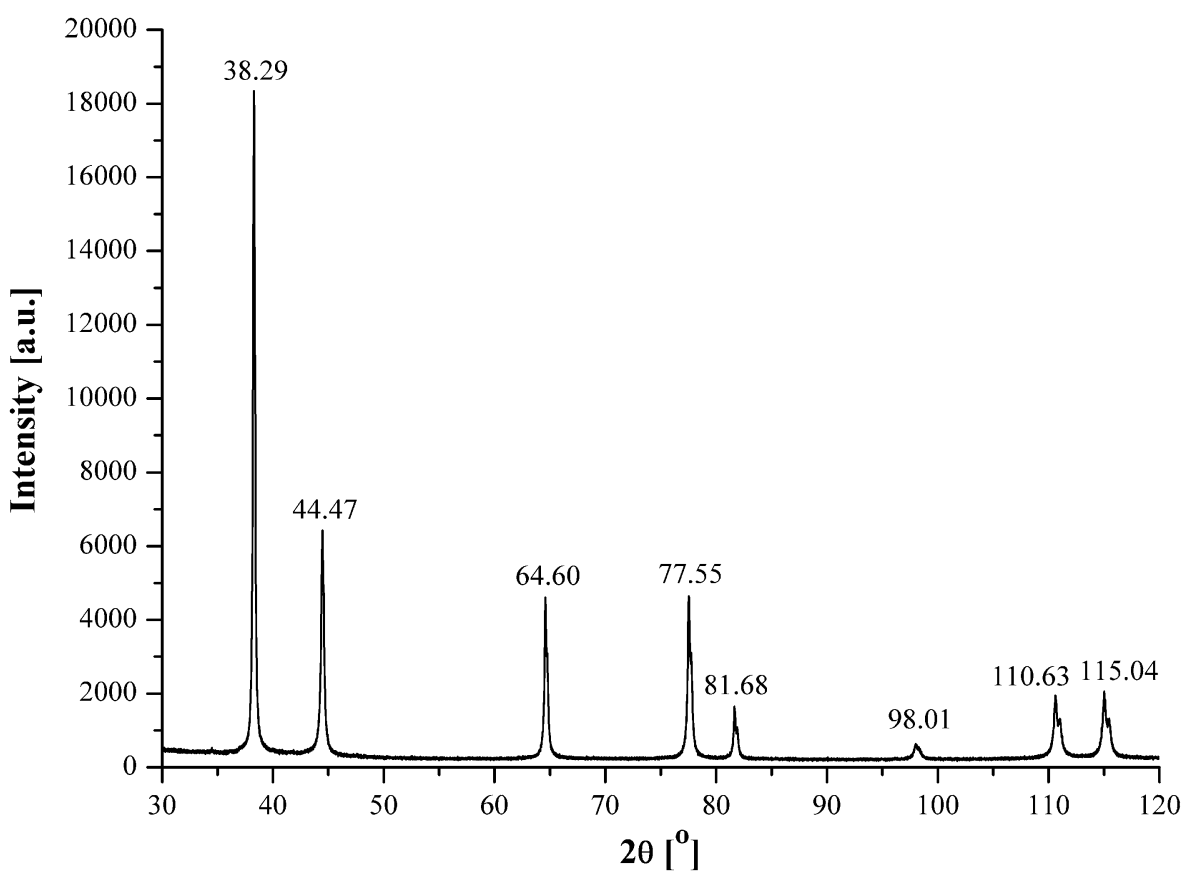

(h k l): (111), (200), (220), (311), (222), (400), (331), (420) respectively. Thus, the XRD diffractograms confirmed the unit crystal cell of face-centered-cubic Ag in nanoparticles (JCPDS file No. 04-0783). No peaks of other impurity crystalline phases have been detected. On the diffractogram specific peak for silver oxides appearing at around $2 \theta=33^{\circ}$ is not present, which imply that the silver nanoparticles are free from silver oxide. The result indicates that the as synthesized product is composed of high purity silver nanoparticles.

\subsection{The morphology of silver nanoparticles}

Depend on the synthesis conditions Ag nanoparticles can be obtained with various sizes and shapes. In order to investigate the morphology of the particles sophisticated microscopic analyses were applied. The TEM images of silver nanoparticles indicates that the nanoparticles are more or less spherical (Fig. 4) and the average diameter ranges from 15 to $50 \mathrm{~nm}$ (Fig. 5). Based on the TEM images nanoparticle diameter distribution have been determined using Aphelion Lab 4.3.2. The resulting distribution chart is shown in Fig. 5. And reveals wide and featureless distribution characteristics. It was found that $53 \%$ of the Ag particles have diameter less than or equal to $25 \mathrm{~nm}$, and about $22 \%$ of nanoparticles have diameter approx. $15 \mathrm{~nm}$.
Fig. 4 TEM images of silver nanoparticles at different magnifications
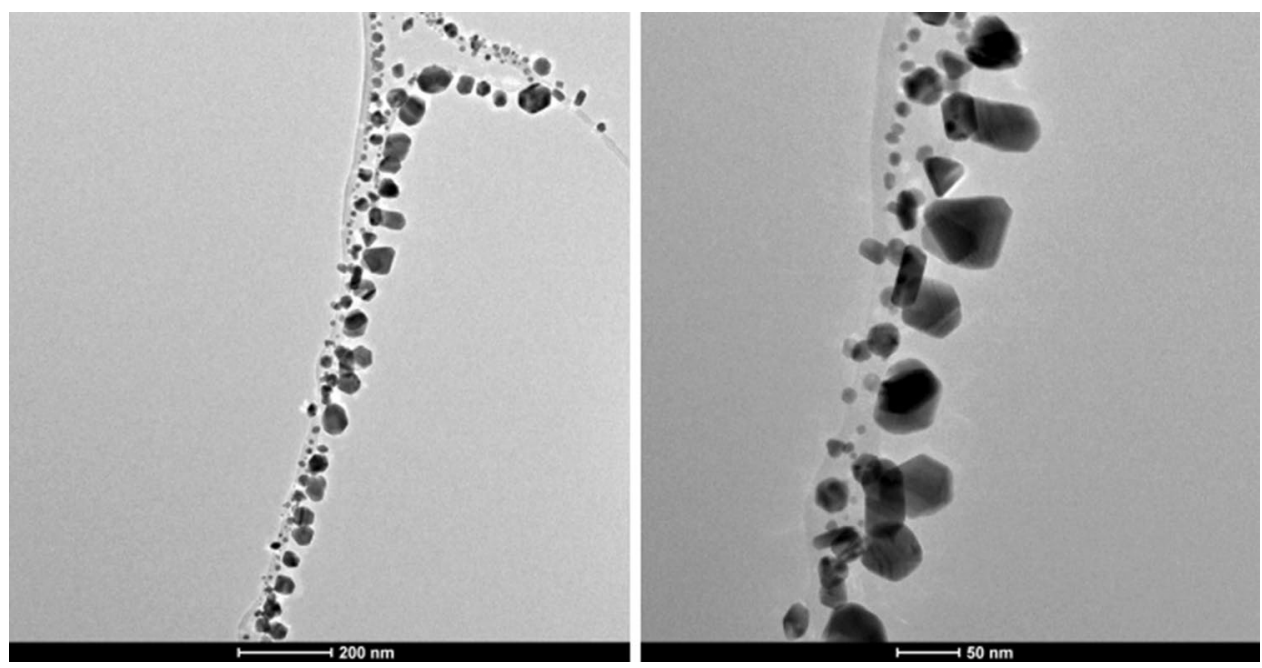


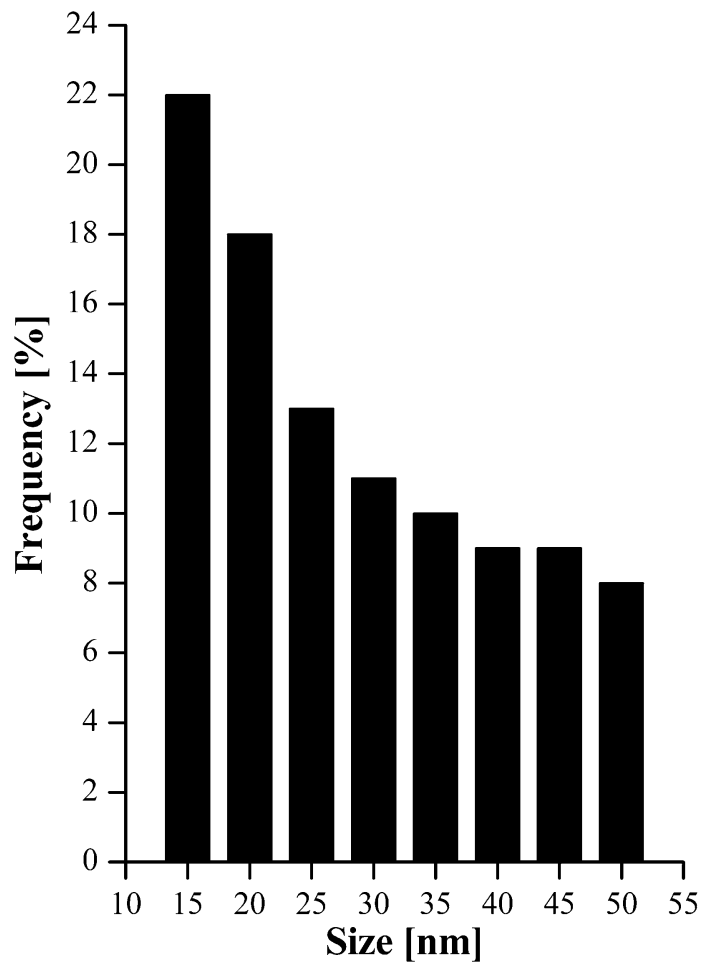

Fig. 5 Silver nanoparticle diameter distribution based on the TEM imaging

\subsection{The polymer surface covered with silver or silver nanoparticles}

The surface morphology of polymer covered with silver or silver nanoparticles were examined using scanning electron microscope (SEM) and atomic force microscope (AFM).

The SEM images of polymer surface with silver contact revealed a ball-type morphology (Fig. 6a, b). Spherical particles of silver covered polymer have diameter ranging from 14 to $112 \mathrm{~nm}$, with an average of $25 \mathrm{~nm}$ (Fig. 7). The distribution chart show halve of the bell-shaped distribution curve. The missing halve of the distribution curve can't be resolved due to resolution of SEM images and gives extremely high count bar at the low end of the distribution. The Fig. 6c, d show micron-sized flacks of agglomerated silver nanoparticles. The film is substantially different from the parent compound. The flacks of metal obscure the initial shape of spherical polymer particles.

Based on the results of AFM analysis (Fig. 8) it was found that the surface of polymer pellets with silver nanoparticles (Fig. 8b, d) is more homogeneous than, the surface of polymer pellets with evaporated silver (Fig. 8a, c). Metallic layer on the polymer resulting from nanosilver evaporation seems to be microcrystalline or even amorphous, while the layer obtained from evaporation of silver is typical polymorphic metal layer.
Fig. 6 SEM images of polymer surface covered with a silver- $\times 50,000, \mathbf{b}$ silver- $\times 350,000$, c silver nanoparticles- $\times 50,000, \mathbf{d}$ silver nanoparticles $-\times 350,000$ (a)

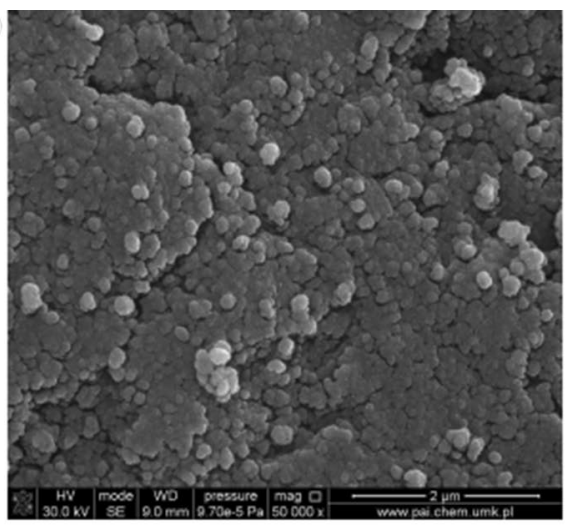

(c)

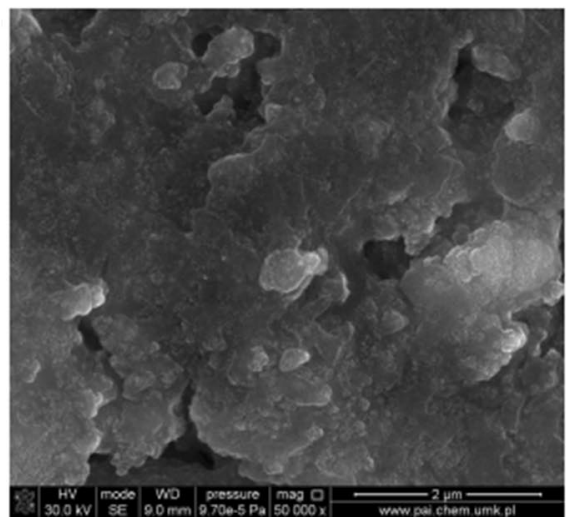

(b)

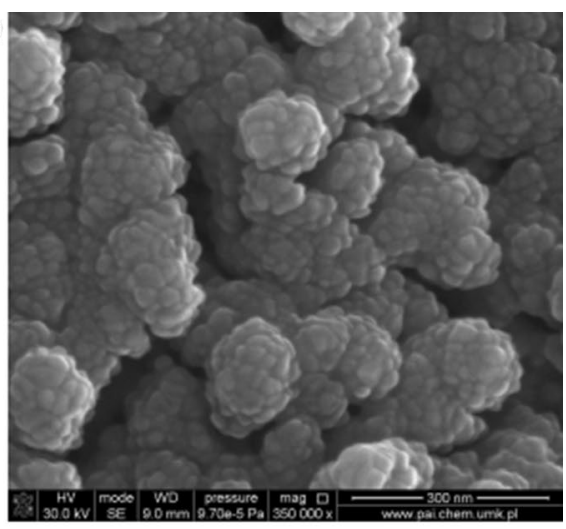

(d)

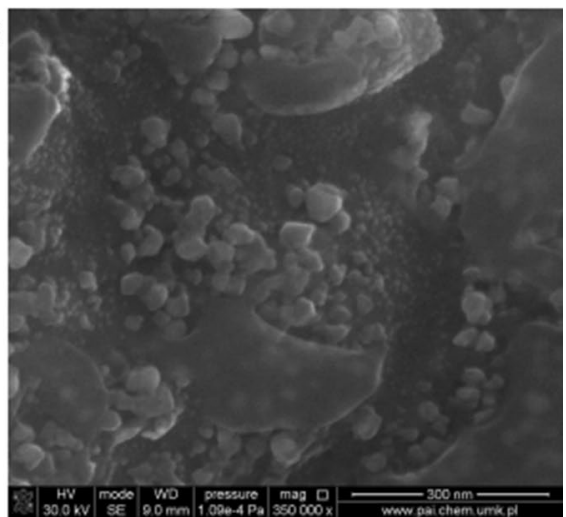




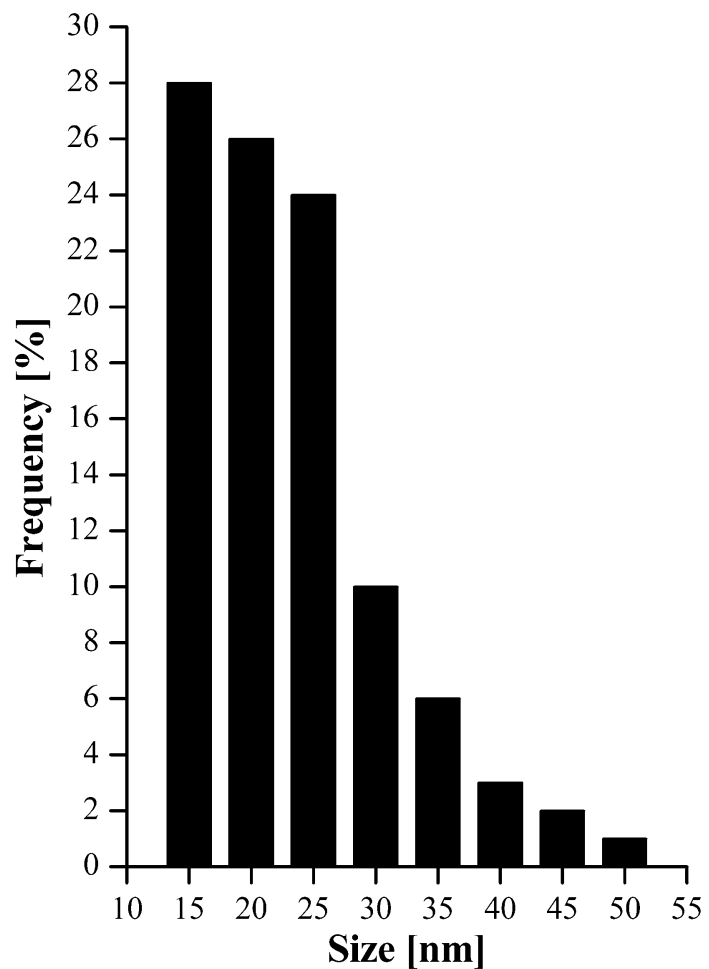

Fig. 7 The particle size distribution for the polymer with silver

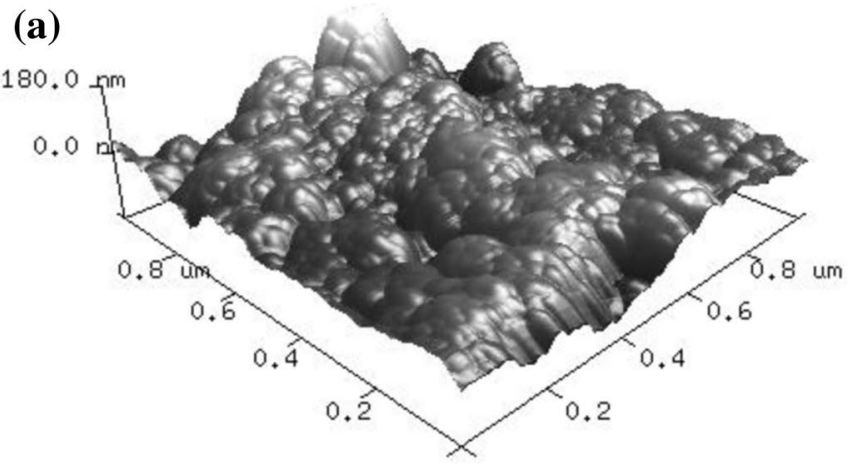

(c)

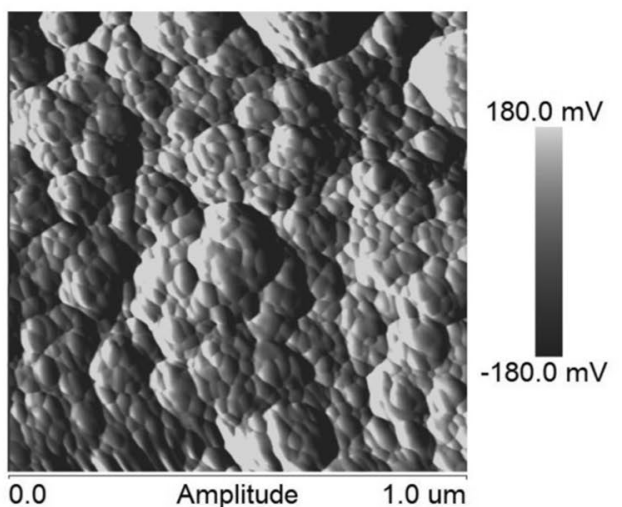

\subsection{Current-voltage characteristics}

Important information on the electrical properties of the system were obtained from the measurements of current-voltage (i-v) characteristics of the heterojunction. The intrinsic conductivity of a given heterojunction $\left(\sigma_{\mathrm{dc}}\right)$ was calculated according to the formula (1):

$\sigma_{d c}=\left(\frac{\partial I}{\partial V}\right)_{T} \frac{d}{S}, \quad\left(\Omega^{-1} \mathrm{~m}^{-1}\right)$

where: $I$-the current [A], $V$-the voltage [V], $d$-thickness of polymer specimen $[\mathrm{m}], s$ - the surface of electrode $\left[\mathrm{m}^{2}\right]$.

The Activation energy of conduction $\left(E_{A}\right)$ was determined by linear regression analysis of linearized form of Arhenius type of $\sigma_{\mathrm{dc}}$ dependence on temperature in:

$\operatorname{Ln}\left(\sigma_{d c}\right)=\operatorname{Ln}\left(\sigma_{0}\right)+\frac{E_{A}}{k_{B}} \cdot \frac{1}{T}$

where: $\sigma_{0}$ is the temperature independent part of the conductivity; $E_{A}$ denotes the activation energy of conduction $(\mathrm{eV})$, $k_{B}$ is the Boltzman's constant and $T$ is absolute temperature (K).

During the preliminary tests it was found that the $\mathrm{i}-\mathrm{V}$ characteristics of the $\mathrm{Ag} /$ polymer heterojunction varies with time. To understand the phenomena the $\mathrm{i}-\mathrm{v}$ characteristics

(b)

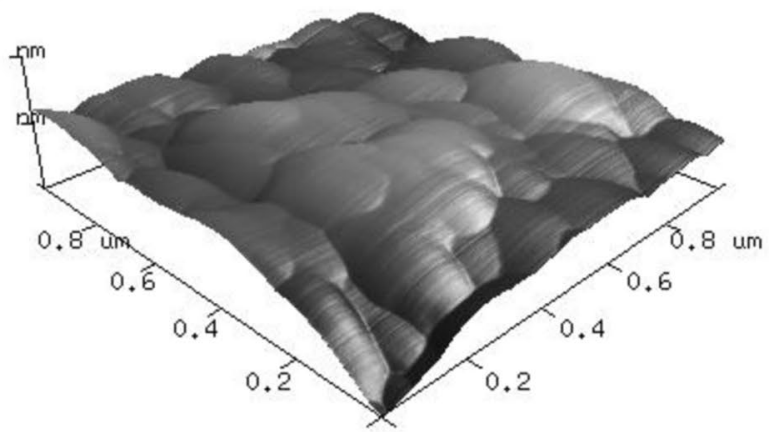

(d)

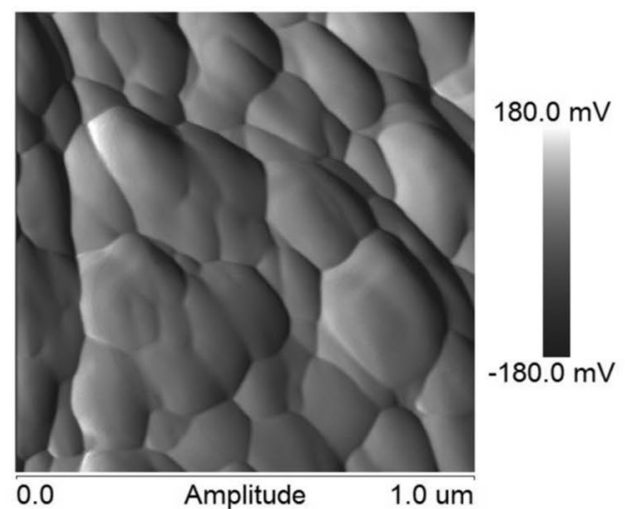

Fig. 8 AFM images of the surface of polymer pellets with evaporated a silver-3D, b silver nanoparticles-3D, $\mathbf{c}$ silver-2D, $\mathbf{d}$ silver nanoparticles-2D 
was first measured immediately after the evaporation of contacts and then, the measurements were repeated several times during following $216 \mathrm{~h}$. It was found that $\sigma_{\mathrm{dc}}$ increases in time, and then stabilizes after about $100 \mathrm{~h}$. At first it was assumed that $\mathrm{Ag}$ atoms diffuse into the polymer establishing metallic domains that increase electrical conductivity. To clarify this issue, a series of measurements was taken in longer time scale. The overall picture of $\sigma_{\mathrm{dc}}$ variation in time is depicted in Fig. 9. However it was also found that the samples having contacts made using normal silver showed perfectly ohmic behavior of the interface up to $96 \mathrm{~h}$, after that $i-v$ plots started to vary from linear. On the other hand corresponding contacts made using silver nanoparticles showed nonlinear $\mathrm{i}-\mathrm{v}$ characteristics in whole considered range of time. The data could be fitted to Moot-Gurney law, however the application of this model is not justified in this case. The Moot-Gurney law requires three limitations, that are not fulfilled in our heterojunction. The model assumptions are: (1) the device is free of energetic traps; (2) there are no diffusion involved; (3) electric field at the injection contact is negligible. Investigated device fails in all three points, so despite curve similarity model could not be applied. In this context the intrinsic conductivity was estimated from linear part of i-v plot at higher electric field. Activation energy of conduction $\left(E_{A}\right)$ determined soon after evaporation for $\mathrm{Ag}$ and nano- $\mathrm{Ag}$ based hetrojunctions was $0.175 \pm 0.01$ and $0.07 \pm 0.01 \mathrm{eV}$ respectively. After $216 \mathrm{~h}$ in ambient conditions the $E_{A}$ of heterojunctions changed and was about $0.085 \pm 0.01$ and $0.137 \pm 0.05 \mathrm{eV}$ for Ag and nano-Ag based

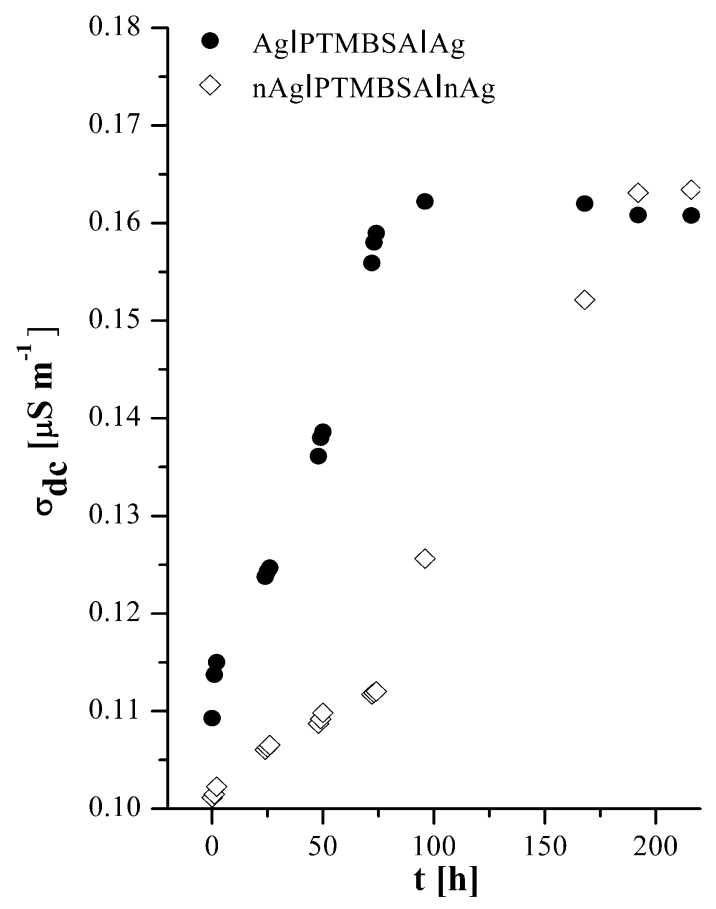

Fig. 9 The change of conductivity over time, $T=298 \mathrm{~K}$ heterojunctions respectively. Additionally, seasoning of both kind of samples results in the increase of $\sigma_{\mathrm{dc}}$. It can be seen from the plots in Fig. 9, where the plot representing Ag contact has sharp slope and reaches the plateau after about $80 \mathrm{~h}$, while plot corresponding to nano-Ag based contact shows moderate increase and reaches the plateau after $170 \mathrm{~h}$. In former case, this behavior is with agreement with initial assumption of metal diffusion from the contact to polymer. Decrease of $E_{A}$ is associated with increasing of doping level, however, in this case silver atoms do not change their oxidation state so they do not dope polymer backbone. The reasonable explanation in this context is the formation of metallic domains within the polymer matrix. Mixed semiconductor and metal conduction of the polymer results in increase of $\sigma_{\mathrm{dc}}$ with simultaneous decrease of $E_{A}$. On the contrary, hetorojunction formed of silver nanoparticles contain large number of different interfaces, both between different nanoparticles and between the particles and organic material. The exceptional behavior coupling the increase of $\sigma_{\mathrm{dc}}$ accompanied with increase of $E_{A}$ is not easy to explain. However, it proves that in contrast to contact made of bulk silver, there is no direct diffusion of metal atom in to polymer. Perhaps entire nanoparticles migrate to polymer moving the contact boundary and decreasing semiconductor layer thickness. This hypothesis could be supported by slower $\sigma_{\mathrm{dc}}$ increase, that can be seen from the plots in Fig. 9. Large, compared to single atoms, nanoparticles migrate slowly. In this context the increase of $\sigma_{\mathrm{dc}}$ can be explained by the decrease of organic layer width, while the increase of $E_{A}$ can be assigned to modification of the interface boundary conditions. At this stage, however detailed interpretation of the phenomenon is premature, more detailed study, including heterojunctions obtained by other metal nanoparticle is necessary to gain the deeper insight in to this new phenomenon.

Current-voltage characteristics presented in Fig. 10 reflect the difference in the plot shapes with respect to difference in contact material. Measured at temperature 298, $308,318 \mathrm{~K}$ for the heterojunctions Ag/polymer shown in Fig. 10a reflect linear relationship of i-v The relationship has a similar shape as the dependence obtained for the pellets of the polymer with the gold electrodes (Au/polymer) [24]. The $\mathrm{i}-\mathrm{v}$ characteristics measured for the heterojunction formed by nano-Ag in the same conditions shows nonlinear plots (Fig. 10b).

Table 1 include the data of dc-conductivity for investigated materials. In both cases, we observe the conductivity growth as the temperature increases.

Further analysis was performed using Simmons' equation at different temperatures (the current density as a function of the electric field strength):

$J=e \mathrm{~N}\left(\mathrm{E}_{F}\right) \mu F \exp \left(-\frac{\phi}{k_{B} T}\right) \exp \left(\frac{\beta F^{0.5}}{k_{B} T}\right)$ 

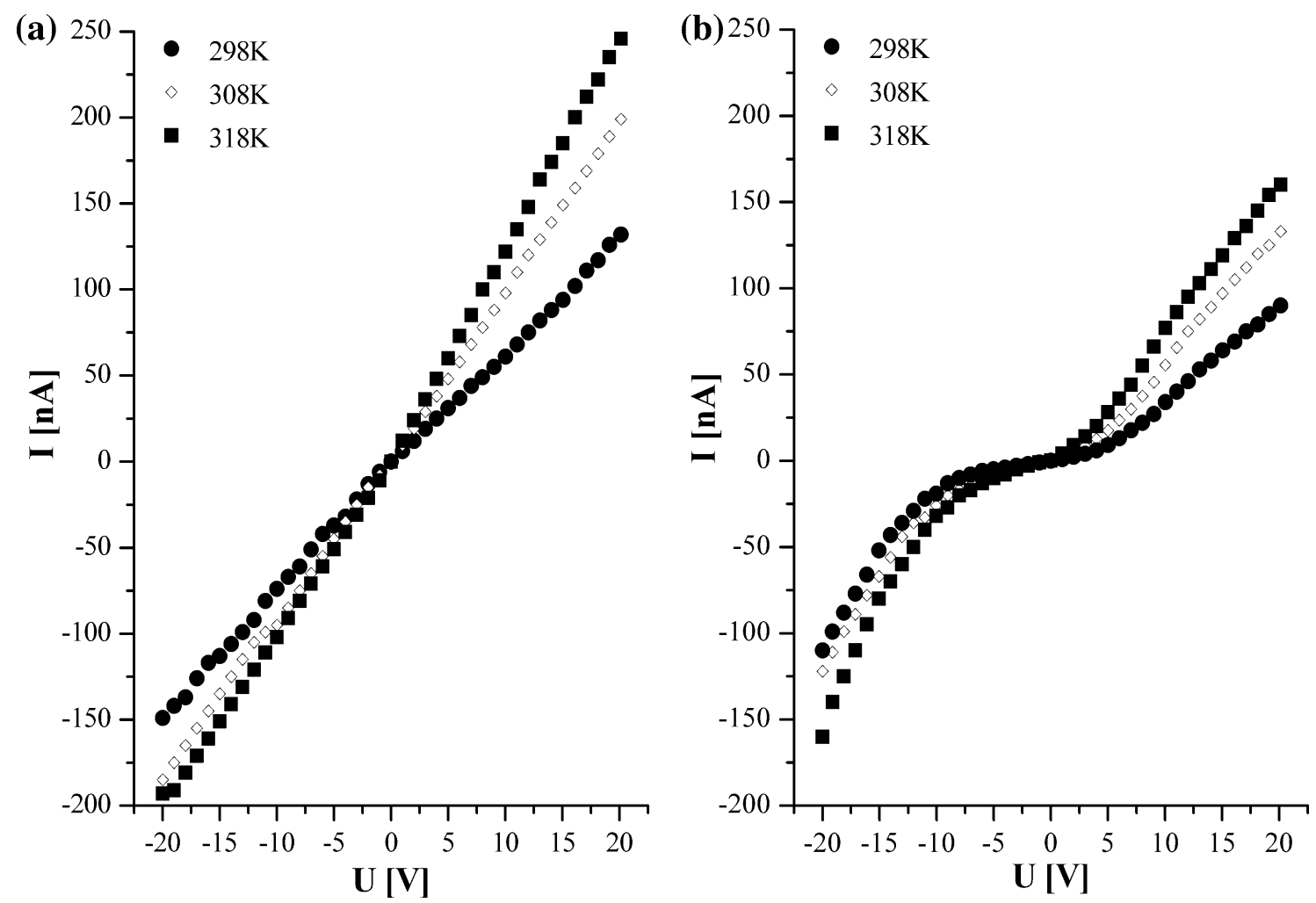

Fig. 10 Current-voltage characteristics for a Ag|PTMBSA|Ag, b nAg|PTMBSAlnAg

Table 1 Electrochemical properties of AglPTMBSAlAg and nAg|PTMBSAlnAg

\begin{tabular}{lll}
\hline Property & $\sigma_{\text {AglPTMBSA|Ag }}$ & $\sigma_{\text {nAglPTMBSAlnAg }}$ \\
\hline$\sigma_{298}\left[\mu \mathrm{S} \mathrm{m}^{-1}\right]$ & 0.1608 & 0.1634 \\
$\sigma_{308}\left[\mu \mathrm{S} \mathrm{m}^{-1}\right]$ & 0.2433 & 0.2651 \\
$\sigma_{318}\left[\mu \mathrm{S} \mathrm{m}^{-1}\right]$ & 0.2863 & 0.3590 \\
\hline
\end{tabular}

where: $J$-the current density [A m ${ }^{-2}$ ], $e$-the elementary charge $\left(1.602 \times 10^{-19} \mathrm{C}\right), \mathrm{N}\left(\mathrm{E}_{F}\right)$ - the effective density of states at the Fermi level of the semiconductor $\left[\mathrm{m}^{-3}\right], \mu-$ the charge carrier mobility $\left[\mathrm{m}^{2} \mathrm{~V}^{-1} \mathrm{~s}^{-1}\right], F$-the electric field strength $\left[\mathrm{V} \mathrm{m}^{-1}\right], \varphi$-the potential energy barrier $[\mathrm{eV}]$, $k_{B}$-the Boltzmann constant $\left(8.618 \times 10^{-5} \mathrm{eV} \mathrm{K}^{-1}\right), T$ - the temperature $[\mathrm{K}], \beta-\mathrm{a}$ constant.

This equation can be linearized to form:

$\ln \frac{J}{F}=\ln \sigma^{0}-\frac{\phi}{k_{B} T}+\frac{\beta}{k_{B} T} F^{0.5}$

where $\sigma^{0}=e \mathrm{~N}\left(\mathrm{E}_{F}\right) \mu\left(\Omega^{-1} \mathrm{~m}^{-1}\right)$.

The analysis of the correlation between $\ln (J / F)$ and $F^{0.5}$ at a particular temperature allows determination of the slope and $\beta$ parameter values (Fig. 11). Further analysis is possible only when the slope value is higher than 0 , i.e. for the
nAglPTMBSAlnAg system. The $\beta$ constant has no physical significance. Therefore, correlation allowing for the assessment of the potential energy barrier and $\sigma^{0}$ using linear regression analysis needs to be used.

$\lim _{F \rightarrow 0}\left(\ln \frac{J}{F}\right)_{T=\text { const }}=\ln \sigma^{0}-\frac{\phi}{k_{B} T}$

The mathematical procedure presented above was used to calculate $\varphi$ and $\sigma^{0}$ values. Results of calculations are listed in the Table 2.

\section{Conclusions}

Electrical properties of the heterojunctions made of new conducting polymer PTMBSA and silver depend on the source of silver used to make metal contacts. The bulk silver lumps evaporate in vacuum to form on the polymer polymorphic crystalline layer that form ohmic contact. In contrast nanosilver metallic layer formed from nanosilver have rather amorphous structure and create rectifying contact with polymer.

As it is shown in the microscopy images, despite the application of the same deposition method the electrodes made of silver and nanosilver have different morphologies. 

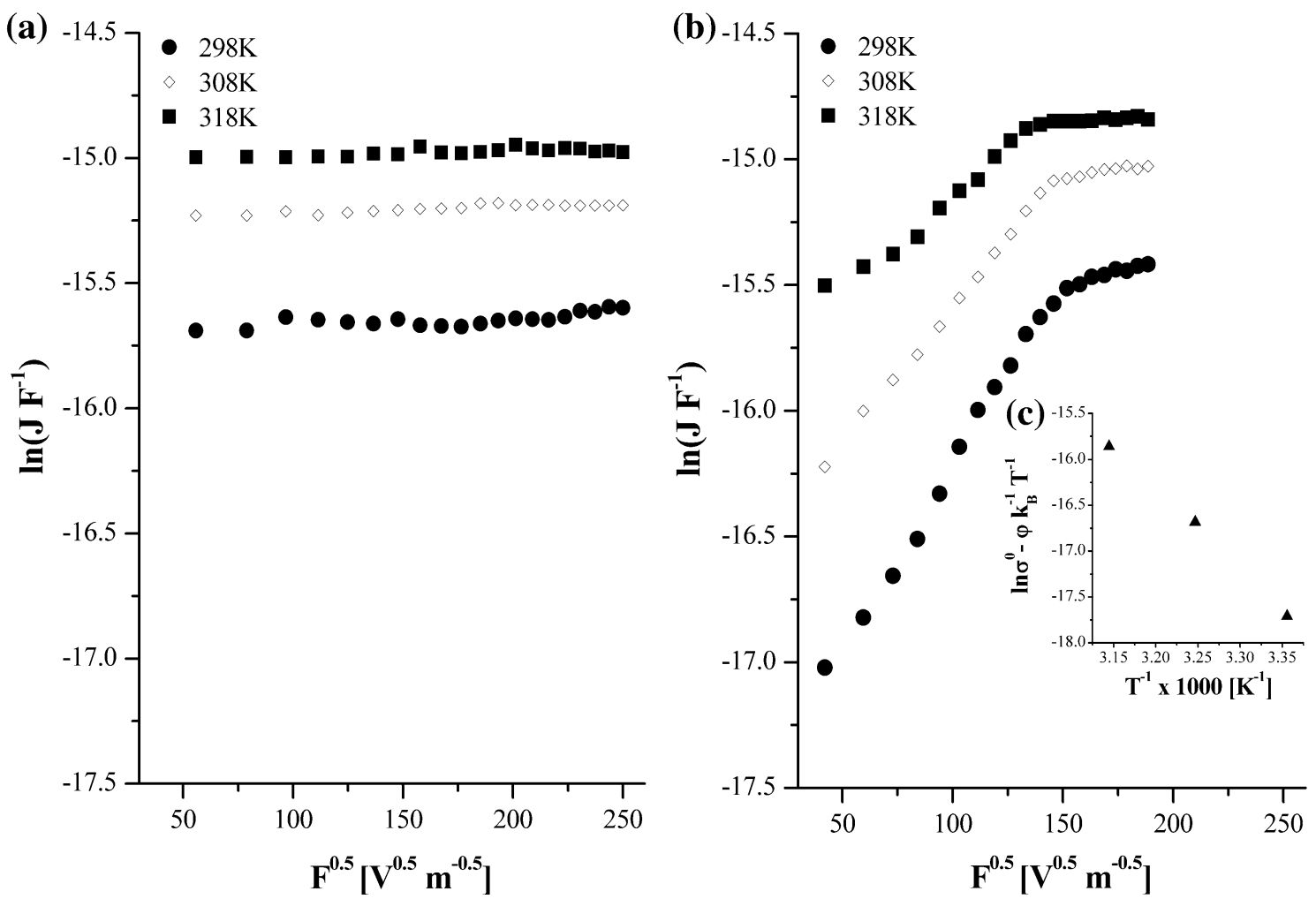

Fig. 11 a The plot of $\ln (J / F)$ vs. square root of applied electric field for AglPTMBSAIAg, b The plot of $\ln (J / F)$ vs. square root of applied electric field for $\mathrm{nAg} \mid \mathrm{PTMBSA} \operatorname{lnAg}, \mathbf{c}$ The plot of $\ln \sigma^{0}-\frac{\phi}{k_{B} T}$ versus $1 / T$

Table 2 Results of the $\mathrm{I}(\mathrm{V})$ analysis of polymer film according to the Simmons equation

\begin{tabular}{ll}
\hline Property & nAglPTMBSAInAg \\
\hline$\varphi[\mathrm{eV}]$ & 0.7556 \\
$\sigma^{0} \times 10^{5}\left[\mathrm{~S} \mathrm{~m}^{-1}\right]$ & 1.250 \\
\hline
\end{tabular}

The former adopts the spherical morphology of the polymer material while the latter covers the surface of the polymer pellet in specific-shaped flakes. Another significant finding concern the diffusion of silver in to polymer matrix. It was found during the studies that electrodes made both of silver and nanosilver undergo diffusion resulting in the increase of the polymer conductivity. However the limiting value of the conductivity is in both cases the same and is about $160 \%$ higher than the initial conductivity, the diffusion from the contact made of normal silver occur faster.

Acknowledgements This work was financially supported by research grant ZB 296 from the Department of Chemistry, Nicolaus Copernicus University in Toruń, Poland.
Open Access This article is distributed under the terms of the Creative Commons Attribution 4.0 International License (http://creativecommons.org/licenses/by/4.0/), which permits unrestricted use, distribution, and reproduction in any medium, provided you give appropriate credit to the original author(s) and the source, provide a link to the Creative Commons license, and indicate if changes were made.

\section{References}

1. K. Gurunathan, A.V. Murugan, R. Marimuthu, U.P. Mulik, D.P. Amalnerkar, Electrochemically synthesised conducting polymeric materials for applications towards technology in electronics, optoelectronics and energy storage devices. Mater. Chem. Phys. 61, 173-191 (1999)

2. T.K. Das, S. Prusty, Review on conducting polymers and their applications. Polym. Plast. Technol. Eng. 51, 1487-1500 (2012). doi:10.1080/03602559.2012.710697

3. M. Lutfi Yola, N. Atar, A. Review, Molecularly imprinted electrochemical sensors for determination of biomolecules/drug. Curr. Anal. Chem. 13, 13-17 (2017)

4. J. Yuan, K. Lu, M. Ford, G.C. Bazan, W. Ma, Dual structure modifications to realize efficient polymer solar cells with low fullerene content. Org. Electron. 32, 187-194 (2016). doi:10.1016/j. orgel.2016.02.026

5. N. Atar, T. Eren, M.L. Yola, A molecular imprinted SPR biosensor for sensitive determination of citrinin in red yeast rice. Food Chem. 184, 7-11 (2015). doi:10.1016/j.foodchem.2015.03.065 
6. M.L. Yola, V.K. Gupta, N. Atar, New molecular imprinted voltammetric sensor for determination of ochratoxin A. Mater. Sci. Eng. C 61, 368-375 (2016). doi:10.1016/j.msec.2015.12.057

7. M.L. Yola, T. Eren, N. Atar, A sensitive molecular imprinted electrochemical sensor based on gold nanoparticles decorated graphene oxide: application to selective determination of tyrosine in milk. Sens. Actuators B 210, 149-157 (2015). doi:10.1016/j. snb.2014.12.098

8. M.L. Yola, T. Eren, N. Atar, A molecular imprinted voltammetric sensor based on carbon nitride nanotubes: application to determination of melamine. J. Electrochem. Soc 163, B588-B593 (2016). doi:10.1149/2.0311613jes

9. M.L. Yola, N. Atar, A highly efficient nanomaterial with molecular imprinting polymer: carbon nitride nanotubes decorated with graphene quantum dots for sensitive electrochemical determination of chlorpyrifos. J. Electrochem. Soc. 164, B223-B229 (2017). doi:10.1149/2.1411706jes

10. N. Atar, M.L. Yola, T. Eren, Sensitive determination of citrinin based on molecular imprinted electrochemical sensor. Appl. Surf. Sci. 362, 315-322 (2016). doi:10.1016/j.apsusc.2015.11.222

11. K. Kadac, A. Nowaczyk, J. Nowaczyk, Synthesis and characterization of new copolymer of pyrrole and 3,4-ethylenedioxythiophene synthesized by electrochemical route. Synth. Met. (2015). doi:10.1016/j.synthmet.2015.05.021

12. N. Atar, T. Eren, M.L. Yola, S. Wang, A sensitive molecular imprinted surface plasmon resonance nanosensor for selective determination of trace triclosan in wastewater. Sens. Actuators B Chem. 216, 638-644 (2015). doi:10.1016/j.snb.2015.04.076

13. B. Ertan, T. Eren, İ. Ermiş, H. Saral, N. Atar, M.L. Yola, Sensitive analysis of simazine based on platinum nanoparticles on polyoxometalate/multi-walled carbon nanotubes. J. Colloid Interface Sci. 470, 14-21 (2016). doi:10.1016/j.jcis.2016.02.036

14. M.L. Yola, N. Atar, Electrochemical detection of atrazine by platinum nanoparticles/carbon nitride nanotubes with molecularly imprinted polymer. Ind. Eng. Chem. Res. 56, 7631-7639 (2017). doi:10.1021/acs.iecr.7b01379

15. R.J. Waltman, J. Bargon, A.F. Diaz, Electrochemical studies of some conducting polythiophene films. J. Phys. Chem. 87, 14591463 (1983). doi:10.1021/j100231a035

16. B. Li, S. Santhanam, L. Schultz, M. Jeffries-EL, M.C. Iovu, G. Sauvé, J. Cooper, R. Zhang, J.C. Revelli, A.G. Kusne, J.L. Snyder, T. Kowalewski, L.E. Weiss, R.D. McCullough, G.K. Fedder, D.N. Lambeth, Inkjet printed chemical sensor array based on polythiophene conductive polymers. Sens. Actuators B 123, 651-660 (2007). doi:10.1016/j.snb.2006.09.064

17. U. Mehmood, A. Al-Ahmed, I.A. Hussein, Review on recent advances in polythiophene based photovoltaic devices. Renew. Sustain. Energy Rev. 57, 550-561 (2016). doi:10.1016/j. rser.2015.12.177

18. F. Liao, M.F. Toney, V. Subramanian, Thickness changes in polythiophene gas sensors exposed to vapor. Sens. Actuators B 148, 74-80 (2010). doi:10.1016/j.snb.2010.04.035

19. M. Jaymand, M. Hatamzadeh, Y. Omidi, Modification of polythiophene by the incorporation of processable polymeric chains: Recent progress in synthesis and applications. Prog. Polym. Sci. 47, 26-69 (2015)

20. T. Ahn, B. Choi, S.H. Ahn, S.H. Han, H. Lee, Electronic properties and optical studies of luminescent polythiophene derivatives. Synth. Met. 117, 219-221 (2001). doi:10.1016/ S0379-6779(00)00368-4

21. H. Zhang, L. Hu, J. Tu, S. Jiao, Electrochemically assembling of polythiophene film in ionic liquids (ILs) microemulsions and its application in an electrochemical capacitor. Electrochim. Acta 120, 122-127 (2014). doi:10.1016/j.electacta.2013.12.091

22. L. Angiolini, A. Brazzi, E. Salatelli, K. Van den Bergh, G. Koeckelberghs, Polythiophene diblock copolymer with different solvent affinities of the side-chain substituents: solvatochromism and effect on the electronic conjugation. Macromol. Chem. Phys. 214, 934-942 (2013)

23. T. Ghoos, J. Brassinne, C.-A. Fustin, J.-F. Gohy, M. Defour, N. Van den Brande, B. Van Mele, L. Lutsen, D.J. Vanderzande, W. Maes, Imidazolium-substituted ionic (co)polythiophenes: Compositional influence on solution behavior and thermal properties. Polymer (Guildf) 54, 6293-6304 (2013). doi:10.1016/j. polymer.2013.09.049

24. K. Kadac, E. Olewnik-Kruszkowska, J. Nowaczyk, Polythiophene with ionophore substituent in the side chain. J. Appl. Polym. Sci. 134 (2017). doi:10.1002/app.44372

25. M.R. Pinto, K.S. Schanze, Conjugated polyelectrolytes: synthesis and applications. Synthesis-Stuttgart 9, 1293-1309 (2002)

26. C. Tan, E. Atas, J.G. Müller, M.R. Pinto, V.D. Kleiman, K.S. Schanze, Amplified quenching of a conjugated polyelectrolyte by cyanine dyes. J. Am. Chem. Soc. 126, 13685-13694 (2004). doi:10.1021/ja046856b

27. P. Urbánek, A. Di Martino, S. Gladyš, I. Kuřitka, A. Minařík, E. Pavlova, D. Bondarev, Polythiophene-based conjugated polyelectrolyte: optical properties and association behavior in solution. Synth. Met 202, 16-24 (2015). doi:10.1016/j. synthmet.2015.01.015

28. M. Kane, K. Krafcik, Nanostructured soluble conducting polyaniline produced by emulsion polymerization. Synth. Met 181, 129-135 (2013). doi:10.1016/j.synthmet.2013.08.021

29. R. Liu, Z. Liu, Polythiophene: synthesis in aqueous medium and controllable morphology. Chinese Sci. Bull. 54, 2028-2032 (2009). doi:10.1007/s11434-009-0217-0

30. S.U.N.J. Lee, J.M.I.N. Lee, I.N.W.O.O. Cheong, H. Lee, J.H. Kim, A facile route of polythiophene nanoparticles via $\mathrm{Fe}^{3+}$-catalyzed oxidative polymerization in aqueous medium. Polymer (Guildf) 46, 2097-2107 (2008). doi:10.1002/pola

31. L. Ai, Y. Liu, X.Y. Zhang, X.H. Ouyang, Z.Y. Ge, A facile and template-free method for preparation of polythiophene microspheres and their dispersion for waterborne corrosion protection coatings. Synth. Met 191, 41-46 (2014). doi:10.1016/j. synthmet.2014.02.004

32. J.M. Lee, S.J. Lee, Y.J. Jung, J.H. Kim, Fabrication of nano-structured polythiophene nanoparticles in aqueous dispersion. Curr. Appl. Phys. 8, 659-663 (2008). doi:10.1016/j.cap.2007.04.049

33. A. Gök, M. Omastová, A.G. Yavuz, Synthesis and characterization of polythiophenes prepared in the presence of surfactants. Synth. Met 157, 23-29 (2007). doi:10.1016/j.synthmet.2006.11.012

34. Z. Wang, Y. Wang, D. Xu, E.S.W. Kong, Y. Zhang, Facile synthesis of dispersible spherical polythiophene nanoparticles by copper(II) catalyzed oxidative polymerization in aqueous medium. Synth. Met 160, 921-926 (2010). doi:10.1016/j. synthmet.2010.02.001

35. L. Dai, Y. Xu, J.-Y. Gal, X. Lu, H. Wu, m-Chloroaniline emulsion polymerization, macromolecular chain structure and electrochemical properties. Polym. Int. 51, 547-554 (2002). doi:10.1002/ pi.881

36. J.-M. Yeh, T.-H. Kuo, H.-J. Huang, K.-C. Chang, M.-Y. Chang, J.-C. Yang, Preparation and characterization of poly(o-methoxyaniline)/Na+-MMT clay nanocomposite via emulsion polymerization: Electrochemical studies of corrosion protection. Eur. Polym. J. 43, 1624-1634 (2007). doi:10.1016/j. eurpolymj.2007.01.040

37. Y.A. Krutyakov, A.A. Kudrinskiy, A.Y. Olenin, G.V. Lisichkin, Synthesis and properties of silver nanoparticles: advances and prospects. Russ. Chem. Rev. 77, 233-257 (2008). doi:10.1070/ RC2008v077n03ABEH003751

38. K. Kadac, E. Olewnik-Kruszkowska, J. Nowaczyk, Polythiophene with ionophore substituent in the side chain. J. Appl. Polym. Sci. (2016). doi:10.1002/app.44372 